\title{
Normas de Concretude para 909 Palavras da Língua Portuguesa
}

\author{
Gerson Américo Janczura ${ }^{1}$ \\ Goiara Mendonça de Castilho \\ Nelson Oliveira Rocha \\ Terezinha de Jesus Cordeiro van Erven \\ Tin Po Huang \\ Universidade de Brasília
}

\begin{abstract}
RESUMO - Normas de concretude para 909 palavras foram coletadas utilizando-se uma escala de julgamento com sete pontos, onde cada extremo representava os níveis altamente abstrato ou concreto, respectivamente. Os resultados evidenciaram uma distribuição bi-modal sugerindo que as palavras podem ser classificadas nas categorias concreta ou abstrata. A confiabilidade média das normas foi $r=0,97$. Não foram detectadas influências do sexo, idade, freqüência de ocorrência das palavras em materiais escritos ou tamanho da categoria nos julgamentos de concretude. Os resultados sugerem que a concretude pode ser considerada como um atributo independente das palavras.
\end{abstract}

Palavras-chave: concretude; normas para palavras; imagem mental.

\section{Concreteness Norms for 909 Portuguese Words}

\begin{abstract}
Concreteness norms were collected for 909 words using a seven point judgment scale, where each extremity highly represented the abstract or concrete levels, respectively. Results showed a bi-modal distribution suggesting that words may be classified either as concrete or abstract. The average reliability of the norms was $r=0.97$. Concreteness judgments were not influenced by sex, age, word written frequency or category size. The results indicate that concreteness may be considered as an independent word attribute.
\end{abstract}

Key words: concreteness; word norms; mental image.

A concretude de uma palavra tem sido associada à experiência ou referência sensorial, seja essa imaginada ou em termos da correspondência com objetos materiais ou pessoas que podem ser experienciados pelos sentidos (Barca, Burani \& Arduino, 2002, Gorman, 1961; Nelson \& Schreiber, 1992; Rocato, 1974; Spreen \& Shulz, 1966).

Paivio, Yuille e Madigan (1968) postulam que o atributo psicológico subjacente à concretude seria a imagem mental, e esse aspecto é comum à maioria das operacionalizações do respectivo constructo. Além disso, estudos têm evidenciado que a concretude pode ser considerada como um atributo independente das palavras (Nelson \& Schreiber, 1992; Paivio, 1968), assim como a freqüência e o comprimento. Metodologicamente, a concretude tem sido mensurada por meio de julgamentos subjetivos nos quais os indivíduos avaliam palavras em uma escala numérica (Nelson \& Schreiber, 1992; Paivio \& cols., 1968; Spreen \& Schulz, 1966; Toglia \& Battig, 1978) ou em uma escala dicotômica, utilizando as categorias concreta ou abstrata (Gorman, 1961).

A influência da concretude de materiais verbais é pungente em tarefas que envolvam a linguagem e a memória. $\mathrm{Na}$ leitura, palavras concretas tendem a ser mais compreensíveis, interessantes e fáceis de memorizar do que palavras abstratas

1 Endereço: Universidade de Brasília, Instituto de Psicologia, PPB, Campus Universitário Darcy Ribeiro, Brasília, DF, Brasil 70910-900. E-mail: janczura@unb.br
(Sadoski, Goetz \& Avila, 1995; Sadoski \& Paivio, 2001). Sentenças concretas são compreendidas mais rapidamente do que sentenças abstratas (Haberlandt \& Graesser, 1985). Os indivíduos respondem mais rapidamente a sentenças concretas do que abstratas quando são solicitados a avaliar a verdade da sentença (Belmore, Yates, Bellack, Jones \& Rosenquist, 1982). Na escrita, a lembrança das relações semânticas entre termos concretos tende a ser mais acurada do que entre palavras mais abstratas (Tirre, Manelis \& Leicht, 1979); além disso, definições para termos concretos tendem a ser mais longas e de melhor qualidade, e os indivíduos relatam usar estratégias imagísticas com esse tipo de material (Sadoski, Kealy, Goetz \& Paivio, 1997).

Na aprendizagem da língua nativa observa-se que palavras concretas são adquiridas com maior facilidade (Gorman, 1961; Paivio, 1963), mais cedo e melhor do que palavras abstratas (Schwanenflugel, 1991; Van Hell \& Candia Mahn, 1997), e na aprendizagem de uma segunda língua, palavras concretas são mais fáceis de adquirir e menos prováveis de serem esquecidas do que palavras mais abstratas (de Groot \& Keijzer, 2000), além da latência para traduzir palavras entre duas línguas ser também influenciada pela concretude (Habuchi, 2003). Esses estudos têm contribuído para questões fundamentais na compreensão da linguagem, como sobre a representação do significado na memória, sobre a memória bilíngüe (Chen \& Leung, 1989; de Groot \& Hoecks, 1995; de Groot \& Root, 1997; La Heij, Hooglander, Kerling \& Van der Velden, 1996) e sobre as implicações instrucionais da 
concretude das palavras nos estágios do desenvolvimento da ortografia (Sadoski, Wilson, Victor, Holcomb \& BoulwareGooden, 2004).

Estudos sobre a memória revelam resultados paralelos da vantagem de palavras concretas em relação às abstratas. A concretude influencia positivamente a decisão léxica (Bleasdale, 1987). Palavras concretas têm um desempenho superior nos paradigmas do reconhecimento (Li, Liu \& Du, 2003) e recuperação livre (Nelson \& Schreiber, 1992; Paivio, 1971, 1986), recuperação com pista extra-lista e intra-lista (Nelson $\&$ Schreiber, 1992). A pesquisa sobre falsas memórias mostra que listas de palavras mais abstratas produzem mais falsas memórias do que listas de palavras mais concretas (PerezMata, Read \& Diges, 2002). Frincke (1968) encontrou que, no paradigma da recuperação, observa-se maior tendência a agrupar itens concretos do que abstratos. Paivio (1976) evidenciou que o efeito da concretude mostrou-se mais pronunciado na recuperação do que no reconhecimento. Por outro lado, os efeitos da concretude são reduzidos se os estímulos são codificados no contexto de prosa (Marschark, 1985; Wattenmaker \& Shoben, 1987) e se as palavras concretas e abstratas estão relacionadas semanticamente (Paivio, Clark \& Khan, 1988; Peterson, 1974). Nesse sentido, Marschark e Hunt (1989) sugerem que o efeito não ocorre no contexto de prosa porque o processamento diferencial das sentenças individuais é, geralmente, negligenciado em favor do processamento relacional entre as sentenças.

Apesar dos efeitos da concretude serem um fenômeno robusto, ainda há muita divergência sobre os seus mecanismos e processos subjacentes. Marschark (1985) e Paivio (1971, 1986) sustentam que palavras mais concretas permitem a geração de imagens mentais que, por sua vez, podem ser utilizadas como recursos extras no desempenho de várias tarefas de linguagem e memória. Por outro lado, a hipótese da disponibilidade contextual assume que informações contextuais de palavras concretas são recuperadas mais facilmente do que das palavras abstratas (Schwanenflugel, Harnishfeger $\&$ Stowe, 1988). Ainda, a hipótese da estrutura associativa defende que as palavras se diferenciam no número de associadas na memória que poderiam ser utilizadas na evocação (de Groot, 1989; Galbraith \& Underwood, 1973).

A teoria do Código Duplo de Paivio (1971, 1986; Sadoski \& Paivio, 2001) é uma das propostas mais aceitas na explicação do fenômeno. Segundo essa teoria, a cognição envolve a atividade de dois sistemas separados mas interconectados: verbal e não-verbal. Palavras mais concretas seriam representadas na memória pelos códigos lexical e sensorial, enquanto as abstratas seriam armazenadas na forma de código lexical. Dessa maneira, palavras concretas teriam mais chances de serem acessadas em função de um maior processamento diferencial do que as palavras mais abstratas.

A inclusão da variável concretude na pesquisa sobre a memória e linguagem, tanto como fator independente como de controle, é extremamente relevante e crítica como evidenciam os resultados apontados acima. Apesar de a literatura disponibilizar normas de concretude para palavras da língua inglesa (e.g., Coltheart, 1981; Paivio \& cols., 1968; Toglia \& Battig, 1978) e italiana (Barca \& cols., 2002), a sua utilização para a língua portuguesa é desaconselhada considerando os efeitos potenciais determinados por diferenças lexicais, semânticas e culturais. O objetivo deste trabalho é oferecer normas de concretude coletadas para 909 palavras da língua portuguesa que poderão ser utilizadas para o desenvolvimento da pesquisa em psicolingüística, memória, linguagem e áreas afins no contexto brasileiro.

\section{Método}

\section{Participantes}

A coleta das normas incluiu 719 participantes, escolhidos por conveniência, cuja participação foi voluntária e anônima. Vinte e dois participantes foram excluídos da análise dos resultados porque dois eram estrangeiros e 22 receberam questionários incompletos. A amostra apresenta os seguintes atributos: $32 \%$ homens e $68 \%$ mulheres; a idade variou de 16 a 60 anos (média $=24,7$ anos, $D P=8,23$ ) sendo que $80 \%$ da amostra têm entre 18 a 31 anos; os participantes são oriundos de 25 unidades da federação e a maioria nasceu no DF $(56,2 \%)$; todos os participantes são estudantes universitários distribuídos em quatro IES do DF, sendo uma pública e três particulares, cujas participações na composição amostral foram respectivamente $49 \%, 21,2 \%, 13 \%$ e 10,3\%; os alunos são oriundos de 37 cursos de graduação, sendo as maiores participações: Psicologia (35,9\%), Letras $(11,9 \%)$, Enfermagem $(11,9 \%)$ e Comunicação Social (10,9\%); o número de semestres cursados na universidade variou de um a 14 (média $=3,73, D P=2,39$ ) e $84,6 \%$ dos alunos estavam matriculados entre o primeiro e sexto semestres.

\section{Materiais}

Foram utilizadas 909 palavras da língua portuguesa sem critérios específicos de seleção. Algumas palavras estão presentes em outras normas (Oliveira, 2001; Stein, Feix \& Rohenkohl, no prelo) e a sua inserção teve por objetivo ampliar o conhecimento sobre as características das mesmas. As palavras foram organizadas aleatoriamente em seis blocos, sendo que três continham 151 palavras e os demais incluíam 152 palavras. O conjunto de palavras está composto pelas seguintes categorias gramaticais: 653 substantivos $(71,8 \%)$, 94 adjetivos (10,3\%), 24 verbos $(2,6 \%)$ e dois advérbios $(0,2 \%)$; as demais palavras pertencem a duas ou mais categorias gramaticais, a saber: 67 substantivos/adjetivos $(7,4 \%)$, 60 substantivos/verbos $(6,6 \%)$, três substantivos/advérbios $(0,3 \%)$ e 3 substantivos/adjetivos/advérbios $(0,3 \%)$.

Cada bloco possuía seis folhas que listavam, em cada página, uma média de 27 palavras. Cada palavra apresentava, à sua direita, uma escala numérica que variava de 1 a 7 , sendo que o valor 1 correspondia ao julgamento "altamente abstrata" e o valor 7 correspondia a "altamente concreta". $\mathrm{O}$ instrumento incluía ainda, no cabeçalho da primeira página, campos destinados à coleta de dados demográficos (idade, sexo, local de nascimento, curso, semestre ou período).

\section{Procedimentos}

A tarefa de cada participante consistiu em julgar a concretude de 151 ou 152 palavras, utilizando uma escala que variava de 1 ("altamente abstrata") a 7 ("altamente concreta"). 
As instruções da tarefa foram semelhantes àquelas utilizadas por Paivio, e cols. (1968), Spreen e Schulz (1966) e Nelson e Schreiber (1992) e são: “Abaixo você encontrará várias palavras. A sua tarefa é julgar o grau de concretude das palavras. Geralmente, palavras que se referem aos objetos, materiais ou pessoas, ou seja, palavras que podem ser experienciadas pelos sentidos são mais concretas do que palavras relacionadas a conceitos abstratos que não produzem uma experiência perceptual. Além disto, é mais fácil e rápido produzir uma imagem mental quando pensamos em palavras mais concretas do que quando pensamos em palavras mais abstratas. Por exemplo, é possível experienciar pelos sentidos e fazer uma imagem da palavra martelo. Por outro lado, é difícil criar uma imagem ou experienciar pelos sentidos a palavra quantidade. Neste caso, a palavra martelo é mais concreta e a palavra quantidade é mais abstrata. Para julgar o grau de concretude você deverá usar uma escala que varia de 1 a 7 , onde o número 7 se refere a palavras altamente concretas e 1 se refere a palavras altamente abstratas. Você poderá usar qualquer número da escala. Para julgar cada palavra circule o número que expressa a sua percepção sobre o grau de concretude da palavra. Alguma dúvida?"

Os dados foram coletados em grupos, em uma única sessão, e cada grupo recebia um número semelhante de cada uma das seis versões do instrumento. Isso garantiu que todas as palavras fossem avaliadas por cada grupo de participantes. No início da sessão, o experimentador lia em voz alta as instruções, que eram seguidas de esclarecimentos sobre eventuais dúvidas dos participantes. O tempo para execução da tarefa era livre, mas observou-se que, em média, os participantes levaram 15 minutos para finalizá-la. Cada versão do instrumento foi respondida por, pelo menos, 100 participantes (média $=112, D P=11,51$ ).

\section{Resultados}

A concretude média de cada palavra foi calculada com base em uma amostra mínima de 92 e máxima de 124 julgamentos, excetuando a palavra "diversão", que teve 202 julgamentos porque foi, equivocadamente, incluída em duas versões do instrumento. Foram excluídas das médias as respostas em branco ou respostas múltiplas para a mesma palavra produzidas pelo mesmo participante. Isso determinou que o julgamento médio, para algumas palavras, fosse inferior a 100 casos.

A Tabela 1, em anexo, apresenta a concretude média e o desvio padrão dos julgamentos para cada palavra. A distribuição dos julgamentos é bi-modal (assimetria $=0,085$; curtose $=-1,480$ ), como pode ser observado na Figura 1, sendo que as freqüências máximas ocorreram em 2,44 e 6,55-6,69. A distribuição sugere que as palavras abstratas estariam na faixa de julgamentos entre 1,61 a 4,45, e palavras concretas na faixa de 4,47 a 6,93. Adotando-se esses intervalos, obtém-se 479 palavras abstratas $(52,7 \%)$ e 430 palavras concretas $(47,3 \%)$. Entre as palavras mais abstratas estão nada $(1,61)$, consciência $(1,61)$, completude $(1,71)$, plenitude $(1,84)$, conseqüência $(1,89)$ e acalento $(1,89)$. Na categoria de palavras mais concretas encontram-se cadeira $(6,93)$, mesa $(6,89)$, sapato $(6,89)$, prato $(6,88)$, pato $(6,86)$ e cobertor $(6,86)$.

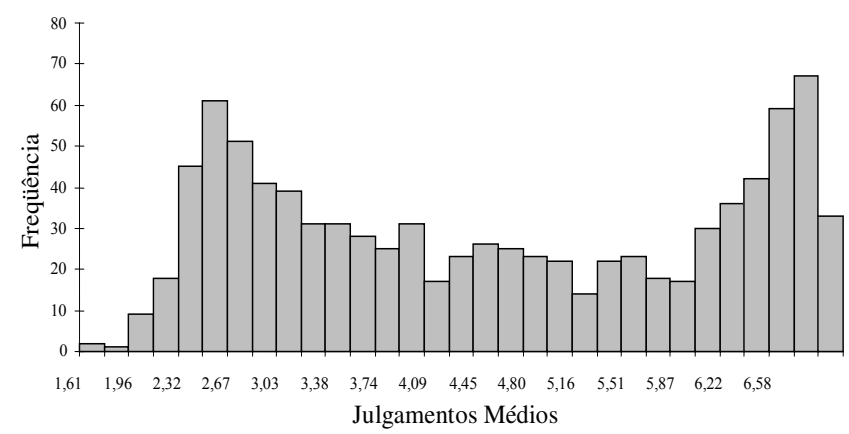

Figura 1. Distribuição de freqüência da concretude.

A confiabilidade interna das normas foi avaliada de várias maneiras. Inicialmente, calculou-se a correlação de Person entre os julgamentos médios das palavras, para cada questionário, dividindo os respondentes em dois grupos aleatoriamente para cada versão do instrumento. As seis correlações obtidas foram significativas ao nível de 0,01 (bilateral) e são, respectivamente, $0,98(n=152), 0,98$ $(n=151), 0,97(n=152), 0,97(n=152), 0,97(n=151)$ e 0,97 $(n=151)$. Esses resultados foram corroborados pelo cálculo de seis ANOVAS, uma para cada questionário, no qual o grupo de respondentes foi, aleatoriamente, dividido em dois subgrupos e a diferença entre as médias dos julgamentos foi comparada. Nenhuma das ANOVAS foi significativa ao nível de significância de $0,05: F(1,300)=0,148, p=0,70$; $F(1,300)=0,309, p=0,579 ; F(1,300)=0,005, p=0,945 ; F(1$, $300)=0,000, p=0,986 ; F(1,300)=0,426, p=0,514$ e, $F(1$, $300)=2,659, p=0,104$.

A freqüência de ocorrência das palavras em materiais escritos não está correlacionada com os julgamentos de concretude. Utilizando-se as freqüências de ocorrência da base de dados LAEL (2006), calculou-se a correlação de Person entre as freqüências de 906 palavras e seus respectivos julgamentos de concretude obtendo-se um $r=-0,009$ $(p=0,78)$. Esse resultado foi corroborado por uma análise da variância para um fator, em que as palavras foram classificadas como abstratas ou concretas utilizando-se as faixas acima descritas, $F(1,905)=0,283, p=0,595$.

A possibilidade de a idade estar associada aos julgamentos de concretude foi avaliada calculando-se a correlação de Person, para cada uma das versões do questionário. Os dados foram, inicialmente, depurados a fim de evitar correlações espúrias determinadas por outliers. Assim, incluiram-se no cálculo de $r$, os participantes cujas idades estavam no intervalo de 16 a 33 anos (média $\pm 1 D P$ ). Nenhuma correlação atingiu o nível de significância de 0,05 , sendo as mesmas, respectivamente, $-0,064(n=89), 0,023(n=103), 0,018(n=96)$, $0,049(n=85), 0,075(n=92)$ e, $0,066(n=106)$.

Investigou-se, também, a influência do sexo nos julgamentos de concretude. Seis análises da variância foram calculadas (uma para cada versão do instrumento), tratando-se o fator sexo como variável inter-sujeitos. Adotando-se o nível de significância de 0,05 , obtiveram-se os seguintes resultados: $F(1,300)=0,454, p=0,501 ; F(1,300)=1,007, p=0,317$; $F(1,300)=2,169, p=0,142 ; F(1,300)=0,214, p=0,644 ; F(1$, $300)=0,492, p=0,484$ e, $F(1,300)=2,095, p=0,149$. Esses resultados revelam que o fator sexo não influenciou os julgamentos de concretude. 
Por fim, verificou-se se o tamanho da categoria poderia estar confundido com a concretude. $\mathrm{O}$ tamanho da categoria compõe um dos aspectos da estrutura das palavras e se refere ao número de associadas produzidas em uma tarefa de associação livre (Nelson, 1981, 1989; Nelson \& McEvoy, 1979). Utilizando o tamanho da categoria das normas de Janczura (1996), calculou-se a correlação entre as duas variáveis para 18 palavras que estavam presentes em Janczura e nas normas de concretude aqui relatadas. Verificou-se que os fatores não estavam correlacionados ( $r=0,29, p=0,24$, bilateral).

\section{Conclusões}

Os julgamentos de concretude evidenciaram que os participantes tenderam a agrupar as palavras em dois grandes grupos: concretas versus abstratas. Apesar de as instruções explicitarem que qualquer grau de concretude pudesse ser atribuído às palavras, os indivíduos tenderam a perceber as palavras de maneira polarizada. A forma da distribuição é semelhante às normas coletadas com palavras da língua inglesa por Nelson e Schreiber (1992). Ou seja, apesar das palavras terem sido avaliadas por falantes de diferentes línguas e contextos culturais, há uma tendência a classificar palavras de diferentes classes gramaticais em duas categorias dominantes.

$\mathrm{O}$ fato de fatores como a freqüência de ocorrência, a idade, o sexo e o tamanho da categoria não terem exercido influência nos julgamentos da concretude sugere, além de corroborar estudos anteriores (e.g., Nelson \& Schreiber, 1992; Paivio, 1968), que a concretude pode ser considerada como um atributo ortogonal das palavras. Essa possibilidade permite que o respectivo fator seja manipulado independentemente em pesquisas que utilizem esse material verbal.

Por fim, acredita-se que as normas de concretude, juntamente com outras normas já coletadas no Brasil, como por exemplo as normas de associação semânticas para conceitos naturais (Janczura, 1996), as normas de tríades para testes de memória (Pompéia, Paes \& Bueno, 2003), as normas de palavras associadas DRM (Stein \& cols., no prelo), as normas de freqüência de ocorrência de Pinheiro (1996) e LAEL (2006), as normas de figuras de objetos comuns (Pompéia, Miranda \& Bueno, 2001) e as normas de associação semântica para palavras contextualizadas (Janczura, no prelo), permitirão ao pesquisador brasileiro avançar no estudo da memória, linguagem e áreas afins utilizando materiais sobre os quais se tem melhor conhecimento podendo, dessa maneira, aprimorar a qualidade e confiabilidade de seus resultados.

\section{Referências}

Bleasdale, F. A. (1987). Concreteness-dependent associative priming: Separate lexical organization for concrete and abstract words. Journal of Experimental Pscyhology: Learning, Memory, \& Cognition, 13, 582-594.

Barca, L., Burani, C. \& Arduino, L. S. (2002). Word naming times and psycholinguistic norms for italian noums. Beharior Research Methods, Instruments and Computers, 34, 424-434.

Belmore, S. M., Yates, J. M., Bellack, D. R., Jones, S. N. \& Rosenquist, S. E. (1982). Drawing inferences from concrete and abstract sentences. Journal of Verbal Learning and Verbal Behavior, 21, 338-351.
Chen, H. C. \& Leung, Y. S. (1989). Patterns of lexical processing in a nonnative language. Journal of Experimental Psychology: Learning, Memory, and Cognition, 15, 316-325.

Coltheart, M. (1981). The MRC Psycholinguistic Database. Quarterly Journal of Experimental Psychology, 33, 497-505.

de Groot, A. M. B. (1989). Representational aspects of word imageability and word frequency as assessed through word association. Journal of Experimental Psychology: Learning, Memory, and Cognition, 15, 824-845.

de Groot, A. M. B. \& Hoecks, J. C. J. (1995). The developmental of bilingual memory: Evidence from word translation by trilinguals. Language Learning, 45, 683-724.

de Groot, A. M. B. \& Root, R. (1997). Word Translation at Three Levels of Proficiency in a Second Language: The Ubiquitous Involvement of Conceptual Memory. Language Learning, 47, 2, 215-264.

de Groot, A. M. B. \& Keijzer, R. (2000). What is hard to learn is easy to forget: The roles of word concreteness, cognate status, and word frequency in foreign-language vocabulary learning and forgetting. Language and Learning, 50(1), 1-56.

Frincke, G. (1968). Word characteristics, associative-relatedness, and the free-recall of nouns. Journal of Verbal Learning \& Verbal Behavior, 7, 366-372.

Galbraith, R. C. \& Underwood, B. J. (1973). Perceived frequency of concrete and abstract words. Memory \& Cognition, 1, 56-60.

Gorman, A. M. (1961). Recognition memory for nouns as a function of abstractness and frequency. Journal of Experimental Psychology, 61, 23-29.

Haberlandt, K. F. \& Graesser, A. C. (1985). Component processes in text comprehension and some of their interactions. Journal of Experimental Psychology: General, 114, 357-375.

Habuchi, Y. (2003). Word Processing in Cross-Language Translation Between Japanese and English by Advanced Second-Language Learners: A Test of the Revised Hierarchical Model. Japanese Journal of Educacional Psychology, 51(1), 65-75.

Janczura, G. A. (1996). Normas associativas para 69 categorias semânticas. Psicologia: Teoria e Pesquisa, 12, 237-244.

Janczura, G. A. (no prelo). Contexto e normas de associação para palavras: a redução do campo semântico, Paidéia.

La Heij, W., Hooglander, A., Kerling, R. \& Van der Velden, E. (1996). Nonverbal context effects in forward and backward word translation: Evidence for concept mediation. Journal of Memory and Language, 35, 648-665.

LAEL (2006). Banco de Português, sub-corpora escrito. http://ww2.lael.pucsp.br/corpora/bp/index.htm, acesso em 07/04/06.

Li, Y., Liu, Y. \& Du, X. (2003). The effect of word concreteness on implicit and explicit memory. Psychological Science, 26(5), 823-828.

Marschark, M. (1985). Imagery and organization in the recall prose. Journal of Memory and Language, 24, 734-745.

Marschark, M. \& Hunt, R. R. (1989). A reexamination of the role of imagery in learning and memory. Journal of Experimental Psychology: Learning, Memory, and Cognition, 15, 710720.

Nelson, D. L. (1981). Many are called but few are chosen: The influence of context on the effects of category size. Em G. H. Bower (Org.), The psychology of learning and motivation, vol. 15 (pp. 129-162). New York: Academic Press. 
Nelson, D. L. (1989). Implicitly activated knowledge and memory. Em C. Izawa (Org.), Current issues in cognitive processes: The Tulane Floweree Symposium on Cognition, (pp. 369-387). Hillsdale: Erlbaum.

Nelson, D. L. \& McEvoy, C. L. (1979). Encoding context and set size. Journal of Experimental Psychology: Human Learning and Memory, 5, 292-314.

Nelson, D. L. \& Schreiber, T. A. (1992). Word concreteness and word structure as independent determinants of recall. Journal of Memory and Language, 31, 237-260.

Oliveira, N. R. (2001). A influência da carga afetiva e do tipo de teste de memória para palavras. Dissertação de Mestrado, Universidade de Brasília, Brasília.

Paivio, A. (1963). Learning of adjective-noun paired-associates as a function of adjective-noun word order and noun abstractness. Canadian Journal of Psychology, 17, 370-379.

Paivio, A. (1968). A factor-analytic study of word attributes and verbal learning. Journal of Verbal Learning and Verbal Behavior, 7, 41-49.

Paivio, A. (1971). Imagery and verbal processes. New York: Holt, Rinehart, and Winston.

Paivio, A. (1976). Imagery in recall and recognition. Em J. Brown (Org.), Recall and recognition (pp. 103-129). London: Wiley.

Paivio, A. (1986). Mental representation: A dual coding approach. New Yor: Oxford University Press.

Paivio, A., Yuille, J. C. \& Madigan, S. A. (1968). Concreteness, imagery, and meaningfulness values for 925 noums. Journal of Experimental monograph Supplement, 76(1, Part 2), 1-25.

Paivio, A., Clark, J. \& Khan, M. (1988). Effects of concreteness and semantic relatedness on composite imagery ratings and cued recall. Memory \& Cognition, 16, 422-430.

Perez-Mata, M. M., Read, J. D. \& Diges, M. (2002). Effects of divided attention and word concreteness on correct recall and false memory reports. Memory, 10(3), 161-177.

Peterson, R. C. (1974). Imagery and cued recall: Concreteness or context? Journal of Experimental Psychology, 102, 841-844.

Pinheiro, A. M. V. (1996). Contagem de freqüência de ocorrência de palavras expostas a crianças na faixa pré-escolar e séries iniciais do $1^{\circ}$. grau. São Paulo: Associação Brasileira de Dislexia.

Pompéia, S., Miranda, M. C. \& Bueno, O. F. A. (2001). A set of 400 pictures standardised for Portuguese: norms for name agreement, familiarity and visual complexity for children and adults. Arquivos de Neuropsiquiatria, 59, 330-337.

Pompéia, S., Paes, A. T. \& Bueno, O. F. A. (2003). Teste de completação de letras: Estímulos para uso no Brasil. Psicologia: Teoria e Pesquisa, 19, 65-73.
Rocato, S. (1974). Concreteness, imagery, meaningfulness and a new index: Values for 100 Italian nouns. Italian Journal of Psychology, 1, 195-209.

Sadoski, M. \& Paivio, A. (2001). Imagery and text: A dual coding theory of reading and writing. Mahwah, New Jersey: Lawrence Erlbaum.

Sadoski, M., Goetz, E. T. \& Avila, E. (1995). Concreteness effects in text recall: Dual coding or context availability? Reading Research Quarterly, 30, 278-288.

Sadoski, M., Kealy, W. A., Goetz , E. T. \& Paivio, A. (1997). Concreteness and imagery effects in the written composition of definitions. Journal of Educational Psychology, 89, 518-526.

Sadoski, M., Willson, V. L., Holcomb, A. \& Boulware-Gooden, R. (2004). Verbal and Nonverbal Predictors of Spelling Performance. Journal of Literacy Research, 36(4), 461-478.

Schwanenflugel, P. J. (1991). Why are abstract concepts hard to understand? Em P.J. Schwanenflugel (Org.), The psychology of word meanings (pp. 223-250). Hillsdale: Lawrence Erlbaum.

Schwanenflugel, P. J., Harnishfeger, K. K. \& Stowe, R. W. (1988). Context availability and lexical decisions for abstract and concrete words. Journal of Memory and Language, 27, 499520.

Spreen, O. \& Schulz, R. W. (1966). Parameter of abstraction, meaningfulness, and pronounciability for 329 noums. Journal of Verbal Learning and Verbal Behavior, 5, 459-468.

Stein, L. M., Feix, L. F. \& Rohenkohl, G. (no prelo). Avanços metodológicos no estudo das falsas memórias: Construção e normatização do procedimento de palavras associadas. Psicologia: Reflexão e Crítica.

Tirre, W. C., Manelis, L. \& Leich, K. L. (1979). The effects of imaginal and verbal strategies on prose comprehension by adults. Journal of Reading Behavior, 11, 99-106.

Toglia, M. P. \& Battig, W. F. (1978). Handbook of semantic word norms. Hillsdale, New Jersey: Laurence Erlbaum.

Van Hell, J. G. \& Candia Mahn, A. (1997). Keyword mnemonics versus rote rehearsal: Learning concrete and abstract foreign words by experienced and inexperienced learners. Language Learning, 47, 507-546.

Wattenmaker, W. D. \& Shoben, E. J. (1987). Context and the recallability of concrete and abstract sentences. Journal of Experimental Psychology: Learning, Memory, and Cognition, $13,140-150$.

Recebido em 24.04.2006 Primeira decisão editorial em 25.09.2006 Aceito em 28.10.2006 
G. A. Janczura e cols

Apêndice

Tabela 1. Concretude média das palavras.

\begin{tabular}{|c|c|c|c|c|c|c|c|c|c|c|c|}
\hline Palavras & $M$ & $D P$ & Palavras & $M$ & $D P$ & Palavras & $M$ & $D P$ & $\begin{array}{l}\text { Palavras } \\
\end{array}$ & $M$ & $D P$ \\
\hline Abacaxi & 6,66 & 0,98 & Aroma & 2,79 & 1,75 & Borrar & 3,83 & 1,63 & Chorar & 4,32 & 1,84 \\
\hline Abençoar & 2,24 & 1,53 & Arquiteto & 5,33 & 1,78 & Botão & 6,26 & 1,45 & Choro & 4,53 & 1,94 \\
\hline Abertura & 3,21 & 1,64 & Arrependimento & 1,95 & 1,46 & Branco & 5,02 & 1,99 & Chuchu & 6,74 & 0,85 \\
\hline Abóbora & 6,55 & 1,09 & Arrepio & 4,10 & 1,73 & Bravo & 3,10 & 1,71 & Chulé & 3,76 & 1,76 \\
\hline Abominável & 2,53 & 1,55 & Artista & 4,54 & 1,81 & Briga & 4,88 & 1,54 & Chute & 5,18 & 1,73 \\
\hline Aborto & 4,53 & 1,98 & Árvore & 6,59 & 1,15 & Brilhante & 3,99 & 2,03 & Chuva & 6,11 & 1,44 \\
\hline Abraço & 4,41 & 1,89 & Asa & 5,93 & 1,55 & Brincadeira & 3,47 & 1,70 & Cidade & 6,15 & 1,29 \\
\hline Acalento & 1,89 & 1,09 & Asfalto & 6,41 & 1,19 & Brinco & 6,51 & 1,12 & Cigarro & 6,75 & 0,72 \\
\hline Acalmar & 2,66 & 1,55 & Áspero & 4,64 & 1,80 & Bule & 6,68 & 0,77 & Cinto & 6,70 & 0,77 \\
\hline Acariciar & 4,24 & 1,95 & Aspirar & 3,23 & 1,97 & Cabeça & 6,72 & 0,88 & Cinza & 4,88 & 1,79 \\
\hline Aceitação & 2,32 & 1,49 & Asquerosa & 2,63 & 1,59 & Cabelo & 6,56 & 0,93 & Circo & 6,15 & 1,43 \\
\hline Acerola & 6,81 & 0,60 & Assalto & 4,25 & 1,72 & Cabra & 6,44 & 1,31 & Ciúme & 2,24 & 1,40 \\
\hline Acidente & 4,39 & 1,99 & Assinatura & 5,61 & 1,57 & Caça & 3,81 & 1,74 & Claridade & 4,67 & 1,62 \\
\hline Acompanhado & 3,79 & 1,85 & Atenção & 2,69 & 1,70 & Cachoeira & 6,45 & 1,10 & Cobertor & 6,86 & 0,51 \\
\hline Aconchego & 2,94 & 1,42 & Atitude & 2,68 & 1,96 & Cachorro & 6,62 & 1,05 & Cocaína & 6,13 & 1,35 \\
\hline Acordar & 4,31 & 1,94 & Atraso & 2,60 & 1,53 & Cadáver & 6,11 & 1,33 & Cochilar & 4,38 & 1,65 \\
\hline Açúcar & 6,41 & 1,05 & Atrito & 3,69 & 1,89 & Cadeado & 6,41 & 1,34 & Colchão & 6,69 & 0,94 \\
\hline Acusação & 2,86 & 1,64 & Aula & 4,19 & 1,85 & Cadeia & 5,94 & 1,54 & Colégio & 6,24 & 1,22 \\
\hline Acusar & 2,89 & 1,41 & Ausência & 2,31 & 1,78 & Cadeira & 6,93 & 0,37 & Cólera & 2,87 & 1,75 \\
\hline Admirável & 2,42 & 1,46 & Automóvel & 6,69 & 0,90 & Caderno & 6,75 & 0,81 & Coleta & 3,38 & 1,74 \\
\hline Adoecer & 3,69 & 1,80 & Autoridade & 3,36 & 1,75 & Café & 6,29 & 1,30 & Colher & 6,63 & 1,10 \\
\hline Adultério & 3,63 & 1,94 & Aventura & 2,18 & 1,17 & Caixão & 6,43 & 1,20 & Colo & 4,11 & 1,88 \\
\hline Advogado & 5,94 & 1,34 & Azul & 4,35 & 2,15 & Caju & 6,65 & 0,97 & Comércio & 5,38 & 1,54 \\
\hline Afeto & 2,24 & 1,33 & Baderna & 3,45 & 1,60 & Calça & 6,57 & 1,05 & Comida & 6,59 & 0,84 \\
\hline Afetuoso & 2,52 & 1,33 & Baixo & 3,59 & 1,90 & Calçado & 6,03 & 1,42 & Comodidade & 2,93 & 1,54 \\
\hline Agasalho & 6,55 & 0,89 & Bala & 6,11 & 1,36 & Calcanhar & 6,35 & 1,26 & Companheiro & 4,23 & 1,87 \\
\hline Agito & 2,76 & 1,57 & Balanço & 4,42 & 1,92 & Calma & 2,37 & 1,59 & Competência & 1,97 & 1,35 \\
\hline Agonia & 2,42 & 1,41 & Baleia & 6,55 & 1,09 & Calo & 5,95 & 1,46 & Completude & 1,71 & 1,23 \\
\hline Agradável & 2,47 & 1,29 & Banana & 6,75 & 0,89 & Calor & 4,03 & 2,05 & Compreensão & 2,20 & 1,47 \\
\hline Agressão & 3,14 & 1,63 & Banco & 6,19 & 1,38 & Calúnia & 2,28 & 1,60 & Comprido & 3,25 & 1,83 \\
\hline Água & 6,42 & 1,04 & Bandeira & 6,42 & 1,07 & Cama & 6,84 & 0,52 & Comunicação & 3,39 & 1,86 \\
\hline Agulha & 6,77 & 0,74 & Bandido & 4,60 & 1,81 & Caminhar & 4,50 & 1,68 & Confiança & 1,94 & 1,46 \\
\hline Aids & 4,72 & 2,08 & Banho & 5,15 & 1,52 & Camisa & 6,73 & 0,97 & Confortável & 2,82 & 1,57 \\
\hline Alegria & 2,44 & 1,55 & Barba & 6,40 & 1,04 & Câncer & 4,58 & 2,00 & Conquista & 3,26 & 1,78 \\
\hline Algodão & 6,64 & 0,77 & Barco & 6,43 & 1,24 & Caneta & 6,72 & 0,96 & Consciência & 1,61 & 1,23 \\
\hline Alguém & 3,23 & 2,02 & Barrento & 4,44 & 1,85 & Cantor & 5,99 & 1,40 & Conseguir & 2,72 & 1,59 \\
\hline Alienado & 2,71 & 1,64 & Barulho & 4,56 & 1,76 & Capaz & 2,61 & 1,73 & Conseqüência & 1,89 & 1,43 \\
\hline Alimento & 5,66 & 1,50 & Base & 3,81 & 2,06 & Carboidratos & 3,80 & 1,96 & Consultório & 5,87 & 1,31 \\
\hline Alívio & 2,86 & 1,62 & Básico & 2,26 & 1,44 & Carência & 2,06 & 1,38 & Contente & 2,95 & 1,72 \\
\hline Almofada & 6,52 & 0,93 & Batalha & 3,89 & 1,89 & Carícia & 3,60 & 1,70 & Continuação & 2,47 & 1,51 \\
\hline Alpinismo & 4,92 & 1,72 & Beatitude & 2,06 & 1,25 & Cárie & 5,86 & 1,50 & Copo & 6,72 & 0,82 \\
\hline Alto & 4,29 & 1,80 & Beber & 5,25 & 1,74 & Carinho & 3,32 & 2,07 & Cor & 3,63 & 2,03 \\
\hline Alucinação & 2,45 & 1,72 & Beijo & 6,02 & 1,39 & Carnaval & 4,57 & 1,76 & Coração & 5,61 & 1,87 \\
\hline Amar & 2,56 & 1,97 & Beleza & 2,94 & 1,82 & Carnívoro & 4,45 & 1,69 & Corajoso & 2,81 & 1,82 \\
\hline Amarelo & 5,23 & 1,90 & Bélico & 3,47 & 1,67 & Carro & 6,85 & 0,52 & Coroa & 6,18 & 1,39 \\
\hline Amargura & 2,41 & 1,50 & Belo & 2,29 & 1,49 & Carta & 6,19 & 1,40 & Corpo & 6,34 & 1,16 \\
\hline Amigo & 3,99 & 2,04 & Bem-estar & 2,68 & 1,49 & Casa & 6,63 & 0,90 & Correnteza & 5,83 & 1,35 \\
\hline Amizade & 2,44 & 1,53 & Benção & 2,94 & 1,80 & Casaco & 6,41 & 1,12 & Corrigir & 3,30 & 1,58 \\
\hline Amor & 2,23 & 1,59 & Benfeitor & 3,00 & 1,58 & Castelo & 6,51 & 0,96 & Corrupção & 3,59 & 1,94 \\
\hline Analgésico & 4,75 & 1,77 & Beterraba & 6,76 & 0,73 & Catástrofe & 4,18 & 1,72 & Cortina & 6,30 & 1,41 \\
\hline Andar & 4,64 & 1,70 & Bíblia & 6,50 & 1,32 & Cedo & 2,93 & 1,56 & Costura & 4,73 & 1,84 \\
\hline Angelical & 2,44 & 1,48 & Bicho & 5,28 & 1,73 & Cega & 3,68 & 1,87 & Couve & 6,44 & 1,19 \\
\hline Angústia & 2,15 & 1,29 & Blusa & 6,73 & 0,71 & Celestial & 2,06 & 1,34 & Cozinha & 6,15 & 1,31 \\
\hline Animação & 3,17 & 1,53 & Bocejo & 5,15 & 1,72 & Cenoura & 6,78 & 0,66 & Criança & 6,57 & 0,92 \\
\hline Animal & 6,28 & 1,28 & Bolo & 6,65 & 0,96 & Cesto & 6,01 & 1,69 & Crime & 3,56 & 1,85 \\
\hline Anormal & 2,75 & 1,73 & Bolso & 5,95 & 1,51 & Céu & 5,18 & 2,14 & Criminoso & 4,74 & 1,81 \\
\hline Ansiedade & 2,42 & 1,65 & Bom & 2,32 & 1,62 & Chá & 6,05 & 1,44 & Cuidado & 2,33 & 1,56 \\
\hline Apagar & 3,77 & 1,76 & Bondade & 2,25 & 1,42 & Chão & 6,23 & 1,27 & Culpa & 2,39 & 1,58 \\
\hline Apoio & 2,52 & 1,59 & Boneca & 6,55 & 1,04 & Chapéu & 6,76 & 0,73 & Cura & 3,27 & 1,75 \\
\hline $\mathrm{Ar}$ & 3,75 & 2,25 & Bonita & 4,15 & 2,06 & Charuto & 6,67 & 0,81 & Curta & 2,96 & 1,73 \\
\hline Aranha & 6,77 & 0,52 & Bonito & 3,59 & 1,92 & Chata & 2,76 & 1,64 & Curtir & 2,56 & 1,52 \\
\hline Ardente & 2,86 & 1,69 & Borboleta & 6,71 & 0,75 & Cheirar & 4,06 & 1,87 & Dança & 5,32 & 1,57 \\
\hline Areia & 6,25 & 1,24 & Bordar & 5,25 & 1,54 & Chinelo & 6,49 & 1,19 & Decadência & 2,28 & 1,24 \\
\hline Armas & 6,42 & 1,05 & Borracha & 6,42 & 1,07 & Chocolate & 6,61 & 1,05 & Decepar & 5,04 & 1,83 \\
\hline
\end{tabular}

Legenda: $M=$ concretude média, $D \mathrm{P}=$ desvio-padrão. 


\begin{tabular}{|c|c|c|c|c|c|c|c|c|c|c|c|}
\hline Palavras & $M$ & $D P$ & Palavras & $M$ & $D P$ & Palavras & $M$ & $D P$ & Palavras & $M$ & $D P$ \\
\hline Decepção & 2,63 & 1,61 & Escola & 5,96 & 1,49 & Fuga & 3,92 & 1,51 & Injustiça & 2,57 & 1,58 \\
\hline Decisão & 2,09 & 1,40 & Escova & 6,75 & 0,70 & Fumar & 5,22 & 1,78 & Inocência & 2,17 & 1,46 \\
\hline Decoração & 4,54 & 1,80 & Escrever & 5,76 & 1,49 & Fundo & 3,66 & 1,70 & Insegurança & 2,16 & 1,40 \\
\hline Dedicado & 2,43 & 1,56 & Escuro & 3,75 & 1,97 & Fúnebre & 2,92 & 1,66 & Inseto & 5,78 & 1,68 \\
\hline Dedos & 6,71 & 0,85 & Esferográfica & 5,40 & 1,91 & Funeral & 4,78 & 1,64 & Instrumento & 5,72 & 1,69 \\
\hline Dedurar & 2,85 & 1,58 & Especialista & 3,62 & 1,79 & Furar & 4,49 & 1,75 & Insuportável & 2,13 & 1,56 \\
\hline Defeito & 2,69 & 1,63 & Esperto & 2,83 & 1,46 & Fúria & 2,45 & 1,60 & Inteligência & 2,31 & 1,77 \\
\hline Defender & 3,03 & 1,63 & Espetar & 4,58 & 1,80 & Furto & 4,90 & 1,80 & Inteligente & 2,51 & 1,77 \\
\hline Defesa & 2,58 & 1,46 & Espirro & 5,19 & 1,77 & Futebol & 5,59 & 1,55 & Intragável & 2,30 & 1,29 \\
\hline Deficiente & 4,20 & 1,85 & Esquecida & 2,65 & 1,37 & Fuzilar & 4,23 & 1,85 & Inverno & 4,17 & 1,68 \\
\hline Deformidade & 3,71 & 1,94 & Esquecimento & 2,34 & 1,50 & Galinha & 6,77 & 0,60 & Ira & 2,71 & 1,71 \\
\hline Degolar & 4,49 & 1,88 & Essencial & 2,25 & 1,71 & Gargalhada & 4,53 & 1,86 & Irritação & 2,86 & 1,74 \\
\hline Deitado & 4,97 & 2,01 & Estado & 3,52 & 1,93 & Garras & 5,25 & 1,80 & Isqueiro & 6,52 & 1,11 \\
\hline Delicada & 2,67 & 1,56 & Estatura & 3,79 & 1,89 & Gasolina & 6,42 & 1,21 & Janela & 6,48 & 1,15 \\
\hline Delícia & 2,95 & 1,79 & Estica & 3,34 & 1,65 & Gato & 6,75 & 0,78 & Jaqueta & 6,73 & 0,72 \\
\hline Delinqüente & 3,31 & 1,61 & Estofado & 6,26 & 1,13 & Geladeira & 6,80 & 0,83 & Jardim & 6,47 & 1,01 \\
\hline Demônio & 2,82 & 1,96 & Estojo & 6,48 & 1,06 & Gelo & 6,38 & 1,13 & Jaula & 6,50 & 0,94 \\
\hline Demora & 2,88 & 1,58 & Estrada & 5,78 & 1,49 & Gentileza & 3,15 & 1,60 & Jesus & 4,30 & 2,50 \\
\hline Dente & 6,75 & 0,70 & Estrelas & 5,76 & 1,57 & Gigante & 4,54 & 1,86 & Jovem & 4,87 & 1,92 \\
\hline Dentista & 6,06 & 1,19 & Estudo & 3,82 & 2,01 & Girafa & 6,78 & 0,70 & Juba & 5,68 & 1,66 \\
\hline Descansar & 4,02 & 1,77 & Estupro & 4,92 & 1,97 & Glória & 2,18 & 1,55 & Julgamento & 3,08 & 1,54 \\
\hline Descanso & 2,86 & 1,74 & Esverdeado & 4,24 & 1,89 & Goiaba & 6,79 & 0,62 & Justiça & 2,61 & 1,86 \\
\hline Descartável & 3,48 & 1,76 & Euforia & 2,45 & 1,45 & Gol & 5,35 & 1,78 & Justo & 2,50 & 1,61 \\
\hline Descaso & 2,41 & 1,43 & Exército & 5,78 & 1,46 & Gola & 6,39 & 1,12 & Juventude & 2,94 & 1,67 \\
\hline Desconhecido & 2,31 & 1,48 & Fada & 3,67 & 2,32 & Gordo & 5,65 & 1,54 & Lã & 6,29 & 1,29 \\
\hline Descontrole & 2,35 & 1,30 & Falador & 3,59 & 1,62 & Gordura & 4,75 & 1,68 & Ladrão & 5,49 & 1,50 \\
\hline Desejo & 2,59 & 1,75 & Família & 4,76 & 1,77 & Gosma & 5,23 & 1,80 & Lagoa & 6,42 & 0,98 \\
\hline Desenho & 4,83 & 1,88 & Famoso & 3,22 & 1,89 & Gostoso & 3,25 & 1,76 & Lágrima & 5,81 & 1,66 \\
\hline Desespero & 2,44 & 1,62 & Farda & 6,04 & 1,34 & Gozador & 2,99 & 1,63 & Lancha & 6,55 & 1,16 \\
\hline Desfazer & 2,73 & 1,50 & Farelo & 5,59 & 1,75 & Gozo & 3,17 & 1,91 & Lanche & 6,03 & 1,26 \\
\hline Desilusão & 1,94 & 1,50 & Farinha & 6,59 & 0,97 & Graça & 2,25 & 1,29 & Lanchonete & 6,19 & 1,19 \\
\hline Desonesto & 2,37 & 1,58 & Faro & 2,78 & 1,69 & Gracioso & 2,17 & 1,30 & Lápis & 6,72 & 0,74 \\
\hline Desperdício & 2,73 & 1,70 & Faxineiro & 6,14 & 1,12 & Grade & 6,28 & 1,25 & Laranja & 6,47 & 1,05 \\
\hline Despreocupação & 2,16 & 1,54 & Fedor & 3,09 & 1,76 & Grama & 6,55 & 0,95 & Lareira & 6,30 & 1,21 \\
\hline Deus & 2,95 & 2,23 & Feia & 2,74 & 1,64 & Grande & 3,76 & 1,96 & Lata & 6,72 & 0,81 \\
\hline Devagar & 3,30 & 1,60 & Felicidade & 2,31 & 1,59 & Gravador & 6,37 & 1,13 & Látex & 5,29 & 1,85 \\
\hline Diabetes & 3,52 & 2,07 & Felino & 4,76 & 1,92 & Gravata & 6,63 & 1,11 & Leão & 6,65 & 0,85 \\
\hline Difícil & 2,65 & 1,53 & Feliz & 2,30 & 1,62 & Gripe & 3,86 & 1,73 & Leite & 6,63 & 0,82 \\
\hline Dinheiro & 5,99 & 1,49 & Feminina & 3,92 & 1,91 & Grito & 4,13 & 1,88 & Lembrança & 2,39 & 1,68 \\
\hline Direção & 2,86 & 1,57 & Férias & 4,36 & 1,81 & Grosseria & 2,42 & 1,35 & Lento & 2,95 & 1,73 \\
\hline Disciplina & 2,62 & 1,52 & Ferida & 5,32 & 1,70 & Grosso & 4,63 & 1,94 & Lerdo & 2,72 & 1,51 \\
\hline Disco & 6,46 & 0,93 & Fermento & 6,05 & 1,30 & Guerra & 3,97 & 1,80 & Lesma & 6,61 & 0,82 \\
\hline Discriminação & 3,18 & 1,77 & Feroz & 3,18 & 1,81 & Guitarra & 6,69 & 0,98 & Letra & 5,25 & 1,63 \\
\hline Distante & 3,56 & 1,79 & Festa & 4,65 & 1,96 & Guloseima & 4,88 & 1,80 & Leveza & 2,44 & 1,31 \\
\hline Diversão & 2,93 & 1,67 & Filhos & 6,21 & 1,23 & Habitante & 5,43 & 1,75 & Liberdade & 2,34 & 1,51 \\
\hline Doação & 3,47 & 1,79 & Fim & 2,54 & 1,80 & Harmonia & 1,96 & 1,39 & Líder & 4,02 & 1,96 \\
\hline Doce & 4,83 & 1,87 & Fina & 3,21 & 1,73 & Haste & 5,46 & 1,93 & Limpar & 4,05 & 1,84 \\
\hline Doença & 3,51 & 1,88 & Fincar & 4,25 & 1,96 & Hecatombe & 2,72 & 2,02 & Linha & 5,36 & 1,92 \\
\hline Dono & 3,38 & 1,92 & Fio & 5,74 & 1,62 & Hino & 4,30 & 1,98 & Livre & 2,40 & 1,57 \\
\hline Dor & 3,08 & 2,00 & Flâmula & 3,90 & 2,27 & Histérico & 3,04 & 1,63 & Livro & 6,69 & 0,77 \\
\hline Dormir & 4,35 & 1,90 & Flexível & 3,19 & 1,68 & Homem & 6,08 & 1,48 & Lixa & 6,57 & 1,02 \\
\hline Doutor & 4,67 & 1,80 & Flor & 6,69 & 0,84 & Homicídio & 4,08 & 1,85 & Lixo & 5,68 & 1,55 \\
\hline Droga & 4,90 & 1,77 & Fobia & 2,29 & 1,62 & Honestidade & 2,56 & 1,74 & Locomoção & 3,91 & 1,87 \\
\hline Duro & 4,69 & 1,81 & Fofo & 3,43 & 1,74 & Honra & 2,15 & 1,44 & Longe & 2,83 & 1,67 \\
\hline Edificante & 2,68 & 1,56 & Fofoca & 3,06 & 1,74 & Horizonte & 3,81 & 2,10 & Louça & 6,16 & 1,46 \\
\hline Edifício & 6,63 & 0,93 & Fogão & 6,65 & 0,97 & Horrível & 2,55 & 1,72 & Loucura & 2,47 & 1,83 \\
\hline Edredom & 6,54 & 1,14 & Fogo & 6,41 & 1,12 & Hospital & 6,56 & 0,85 & Lua & 6,25 & 1,47 \\
\hline Elástica & 3,30 & 1,70 & Fome & 3,14 & 2,04 & Identificação & 2,84 & 1,75 & Luar & 4,78 & 1,91 \\
\hline Elefante & 6,73 & 0,89 & Forçado & 2,37 & 1,32 & Igreja & 6,23 & 1,31 & Lugar & 3,06 & 1,70 \\
\hline Elevado & 2,79 & 1,64 & Formado & 2,90 & 1,79 & Imbecil & 2,30 & 1,50 & Luto & 3,41 & 1,93 \\
\hline Elogio & 2,35 & 1,48 & Forte & 3,53 & 1,70 & Imoral & 2,19 & 1,52 & Luz & 4,75 & 2,05 \\
\hline Emoção & 2,28 & 1,57 & Fracasso & 2,49 & 1,54 & Império & 4,21 & 1,88 & Maçã & 6,84 & 0,49 \\
\hline Empresário & 5,46 & 1,30 & Frágil & 2,95 & 1,57 & Impiedade & 2,44 & 1,45 & Macho & 4,58 & 1,90 \\
\hline Encosto & 4,19 & 2,11 & Fragrância & 3,86 & 1,79 & Impotente & 2,42 & 1,51 & Machucado & 5,11 & 1,69 \\
\hline Energia & 2,96 & 1,65 & Francês & 3,74 & 1,97 & Incentivo & 2,57 & 1,38 & Macio & 3,96 & 1,92 \\
\hline Enfermeiro & 6,13 & 1,38 & Freira & 6,37 & 1,03 & Incômodo & 2,75 & 1,43 & Maconha & 5,86 & 1,67 \\
\hline Engenheiro & 5,50 & 1,73 & Frescor & 2,85 & 1,66 & Independência & 2,53 & 1,60 & Macumba & 2,78 & 1,64 \\
\hline Entusiasmo & 2,43 & 1,71 & Frio & 3,42 & 1,79 & Infância & 4,15 & 1,75 & Madeira & 6,45 & 1,06 \\
\hline Erro & 2,87 & 1,72 & Frustração & 2,39 & 1,38 & Infidelidade & 2,98 & 1,89 & Madura & 3,71 & 1,73 \\
\hline Escalada & 4,21 & 1,88 & Fruta & 6,57 & 0,86 & Injeção & 6,33 & 1,07 & Mãe & 5,95 & 1,55 \\
\hline
\end{tabular}

Legenda: $M=$ concretude média, $D P=$ desvio-padrão. 
G. A. Janczura e cols

\begin{tabular}{|c|c|c|c|c|c|c|c|c|c|c|c|}
\hline Palavras & $M$ & $D P$ & Palavras & $M$ & $D P$ & Palavras & $M$ & $D P$ & Palavras & $M$ & $D P$ \\
\hline Mágoa & 2,50 & 1,58 & Neve & 5,97 & 1,56 & Peso & 3,85 & 1,97 & Responsabilidade & 2,28 & 1,45 \\
\hline Magro & 5,27 & 1,61 & Nicotina & 4,12 & 2,03 & Pêssego & 6,47 & 1,21 & Resto & 2,98 & 1,80 \\
\hline Majestade & 4,33 & 2,10 & Nobreza & 2,50 & 1,56 & Pessoa & 5,36 & 1,85 & Revólver & 6,72 & 0,92 \\
\hline Mal-estar & 2,78 & 1,46 & Noite & 4,77 & 1,87 & Picada & 5,30 & 1,68 & Rigidez & 2,94 & 1,82 \\
\hline Mal & 2,06 & 1,52 & Nojo & 2,36 & 1,58 & Pico & 4,58 & 1,98 & Rio & 6,61 & 0,88 \\
\hline Maligno & 2,53 & 1,69 & Notas & 4,16 & 2,11 & Pires & 6,36 & 1,39 & Riqueza & 3,35 & 1,89 \\
\hline Manga & 6,23 & 1,29 & Novo & 2,85 & 1,79 & Pisar & 4,56 & 1,79 & Riscar & 4,38 & 1,82 \\
\hline Manteiga & 6,48 & 1,04 & Nudez & 5,37 & 1,64 & Planeta & 5,60 & 1,93 & Ritmo & 3,17 & 1,56 \\
\hline Mão & 6,76 & 0,75 & Nutritiva & 3,51 & 1,60 & Plenitude & 1,84 & 1,41 & Rocha & 6,55 & 1,06 \\
\hline Máquina & 5,44 & 1,69 & Nuvens & 5,84 & 1,70 & Pneu & 6,78 & 0,76 & Rodas & 6,50 & 0,96 \\
\hline Mar & 6,63 & 0,74 & Obedecer & 2,54 & 1,58 & Pó & 5,61 & 1,57 & Rodoviária & 6,29 & 1,11 \\
\hline Maracujá & 6,62 & 1,15 & Objetivo & 2,70 & 1,52 & Pobreza & 4,35 & 1,90 & Rosa & 5,90 & 1,53 \\
\hline Maravilhosa & 2,28 & 1,60 & Objeto & 6,11 & 1,93 & Poder & 2,27 & 1,52 & Roubo & 4,51 & 1,81 \\
\hline Marca & 3,62 & 1,89 & Obrigar & 2,46 & 1,45 & Podre & 3,48 & 1,74 & Roupa & 6,33 & 1,16 \\
\hline Marcha & 4,89 & 1,72 & Obstáculo & 3,59 & 1,92 & Poema & 4,27 & 2,17 & Rua & 6,48 & 0,91 \\
\hline Margarina & 6,34 & 1,30 & Ódio & 2,21 & 1,00 & Polícia & 5,94 & 1,52 & Rude & 2,83 & 1,48 \\
\hline Margem & 4,54 & 1,92 & Odor & 3,21 & 1,93 & Policial & 6,44 & 1,07 & Rugido & 3,81 & 1,82 \\
\hline Marido & 5,89 & 1,52 & Olfato & 3,99 & 2,08 & Poltrona & 6,66 & 1,02 & Rugoso & 4,62 & 1,80 \\
\hline Marte & 5,48 & 1,98 & Onça & 6,74 & 0,90 & Poluição & 4,50 & 1,78 & Ruim & 2,53 & 1,65 \\
\hline Masculino & 4,33 & 1,92 & Oprimido & 2,52 & 1,55 & Ponta & 4,80 & 1,83 & Russa & 3,58 & 2,17 \\
\hline Massa & 5,01 & 1,74 & Ordem & 2,35 & 1,47 & Ponte & 6,47 & 1,03 & Sabedoria & 2,33 & 1,65 \\
\hline Mastro & 6,03 & 1,54 & Orgânico & 3,86 & 1,90 & População & 4,96 & 1,70 & Sabor & 3,07 & 1,80 \\
\hline Material & 4,35 & 2,01 & Organização & 2,93 & 1,52 & Porcelana & 5,91 & 1,47 & Saco & 6,12 & 1,32 \\
\hline Médico & 6,18 & 1,30 & Orgulho & 2,44 & 1,47 & Porco & 6,70 & 0,63 & Saia & 6,37 & 1,27 \\
\hline Medida & 2,84 & 1,85 & Ouvinte & 4,03 & 2,01 & Porta & 6,62 & 1,16 & Sala & 5,88 & 1,36 \\
\hline Medo & 2,52 & 1,82 & Ouvir & 3,33 & 1,82 & Prato & 6,88 & 0,52 & Salada & 6,29 & 1,09 \\
\hline Meia & 6,18 & 1,57 & Paciência & 1,92 & 1,20 & Prazer & 3,02 & 1,97 & Salgado & 4,40 & 2,06 \\
\hline Meiga & 2,66 & 1,48 & Pai & 6,11 & 1,43 & Preconceito & 1,97 & 1,22 & Sanduíche & 6,54 & 0,95 \\
\hline Mel & 6,20 & 1,27 & País & 4,35 & 1,98 & Prédio & 6,53 & 1,11 & Sangue & 6,10 & 1,33 \\
\hline Melancia & 6,81 & 0,63 & Paisagem & 4,73 & 1,99 & Preguiça & 3,08 & 1,81 & Sapato & 6,89 & 0,48 \\
\hline Melancolia & 2,23 & 1,40 & Paixão & 2,50 & 1,62 & Prejudicial & 2,64 & 1,43 & Satisfação & 2,35 & 1,53 \\
\hline Melodia & 3,48 & 1,91 & Palácio & 5,75 & 1,70 & Presídio & 6,08 & 1,45 & Saudade & 1,98 & 1,53 \\
\hline Menina & 6,06 & 1,35 & Paladar & 3,40 & 1,84 & Preto & 4,97 & 2,00 & Saudável & 2,94 & 1,72 \\
\hline Mentira & 2,76 & 1,84 & Palheiro & 5,57 & 1,63 & Prisão & 5,45 & 1,87 & Saúde & 3,55 & 1,87 \\
\hline Mesa & 6,89 & 0,60 & Panela & 6,56 & 1,16 & Problema & 2,83 & 1,84 & Seco & 3,88 & 1,98 \\
\hline Metal & 5,57 & 1,66 & Pânico & 2,33 & 1,56 & Professor & 6,11 & 1,20 & Segurança & 3,05 & 1,42 \\
\hline Metrópole & 5,00 & 1,93 & Pano & 6,29 & 1,23 & Profissional & 3,43 & 1,90 & Selva & 5,60 & 1,58 \\
\hline Militar & 5,67 & 1,65 & Pão & 6,79 & 0,59 & Proteção & 2,67 & 1,51 & Selvageria & 2,54 & 1,49 \\
\hline Moda & 2,94 & 1,84 & Paparicar & 2,72 & 1,57 & Prova & 5,51 & 1,62 & Sensação & 2,13 & 1,47 \\
\hline Momento & 2,13 & 1,54 & Papel & 6,64 & 0,87 & Psicólogo & 5,12 & 1,74 & Sentar & 4,93 & 1,87 \\
\hline Monarquia & 3,33 & 1,82 & Paquerar & 3,42 & 1,93 & Pular & 4,93 & 1,74 & Sentimento & 2,21 & 1,69 \\
\hline Montanha & 6,14 & 1,27 & Paraíso & 2,97 & 1,98 & Pulmão & 6,26 & 1,18 & Sepultar & 4,35 & 1,89 \\
\hline Moradia & 4,83 & 1,84 & Paranóico & 2,39 & 1,49 & Pulseira & 6,37 & 1,11 & Ser & 1,98 & 1,56 \\
\hline Morango & 6,63 & 1,18 & Parar & 3,55 & 1,89 & Pureza & 2,19 & 1,45 & Serenidade & 1,92 & 1,29 \\
\hline Mordida & 5,36 & 1,67 & Parede & 6,69 & 0,78 & Quartel & 5,92 & 1,47 & Seringa & 6,83 & 0,58 \\
\hline Morro & 5,90 & 1,43 & Passada & 2,62 & 1,72 & Quarto & 5,91 & 1,54 & Serra & 5,26 & 1,78 \\
\hline Morte & 3,04 & 1,82 & Pássaros & 6,34 & 1,27 & Quebrada & 3,10 & 1,85 & Sexo & 4,77 & 2,05 \\
\hline Mosca & 6,61 & 0,85 & Patas & 6,24 & 1,42 & Queijo & 6,76 & 0,65 & Shopping & 5,93 & 1,49 \\
\hline Moto & 6,66 & 0,88 & Pátio & 5,44 & 1,69 & Quente & 3,76 & 1,92 & Signo & 2,72 & 1,75 \\
\hline Motor & 6,41 & 1,04 & Pato & 6,86 & 0,42 & Raça & 3,54 & 2,03 & Silêncio & 3,20 & 2,16 \\
\hline Móvel & 5,54 & 1,84 & Pátria & 3,12 & 1,99 & Racional & 2,51 & 1,77 & Símbolo & 3,15 & 1,79 \\
\hline Movimento & 3,20 & 1,84 & Pavor & 2,99 & 1,63 & Rádio & 6,72 & 0,76 & Simpatia & 2,45 & 1,48 \\
\hline Mulher & 6,30 & 1,25 & $\mathrm{Paz}$ & 2,23 & 1,71 & Rainha & 5,46 & 1,63 & Soberano & 2,44 & 1,40 \\
\hline Multidão & 5,23 & 1,65 & Pé & 6,69 & 0,91 & Raiva & 2,72 & 1,59 & Social & 2,15 & 1,40 \\
\hline Muro & 6,53 & 1,01 & Pecado & 2,29 & 1,76 & Rancor & 1,98 & 1,41 & Sofá & 6,64 & 1,12 \\
\hline Músculos & 6,23 & 1,08 & Peçonha & 3,32 & 2,03 & Rápido & 3,08 & 1,75 & Sofrimento & 2,48 & 1,50 \\
\hline Música & 4,34 & 2,05 & Pedra & 6,65 & 0,98 & Realização & 2,44 & 1,64 & Sol & 6,07 & 1,42 \\
\hline Nação & 3,53 & 1,81 & Peixe & 6,76 & 0,65 & Reciclagem & 3,95 & 1,84 & Soldado & 6,55 & 0,97 \\
\hline Nada & 1,61 & 1,44 & Pele & 5,79 & 1,80 & Reciprocidade & 2,37 & 1,60 & Solidão & 2,16 & 1,47 \\
\hline Nadar & 5,15 & 1,56 & Peluda & 4,90 & 1,78 & Rede & 5,72 & 1,74 & Som & 3,39 & 1,87 \\
\hline Namorado & 5,42 & 1,76 & Pequena & 3,67 & 1,73 & Rei & 5,00 & 1,66 & Sombra & 3,76 & 1,99 \\
\hline Namoro & 3,97 & 1,77 & Pêra & 6,82 & 0,59 & Relaxamento & 2,36 & 1,40 & Sonhar & 2,28 & 1,56 \\
\hline Nariz & 6,54 & 1,15 & Perda & 2,57 & 1,90 & Remédio & 5,86 & 1,31 & Sonolento & 3,68 & 1,69 \\
\hline Nascimento & 3,67 & 1,96 & Perdoar & 2,41 & 1,59 & Remorso & 2,03 & 1,47 & Sopa & 6,54 & 0,77 \\
\hline Natureza & 4,06 & 2,07 & Perfume & 5,34 & 1,83 & Repolho & 6,69 & 0,83 & Sorriso & 5,02 & 1,97 \\
\hline Navalha & 6,33 & 1,24 & Perigo & 3,17 & 1,59 & Repouso & 3,27 & 1,79 & Sorvete & 6,49 & 1,19 \\
\hline Necessidade & 2,19 & 1,46 & Pernas & 6,58 & 0,88 & Representação & 2,24 & 1,45 & Sossego & 2,32 & 1,36 \\
\hline Negativo & 2,49 & 1,56 & Persiana & 6,65 & 0,88 & Repressão & 2,58 & 1,54 & Status & 2,91 & 1,54 \\
\hline Negro & 5,12 & 1,74 & Pesadelo & 2,84 & 1,82 & Respeito & 2,61 & 1,83 & Suave & 2,59 & 1,35 \\
\hline Nervosismo & 2,57 & 1,61 & Pesca & 4,95 & 1,55 & Respirar & 4,40 & 1,91 & Subir & 3,51 & 1,74 \\
\hline
\end{tabular}

Legenda: $M=$ concretude média, $D \mathrm{P}=$ desvio-padrão. 
Normas de Concretude para Palavras

\begin{tabular}{|c|c|c|c|c|c|c|c|c|c|c|c|}
\hline Palavras & $M$ & $D P$ & Palavras & $M$ & $D P$ & Palavras & $M$ & $D P$ & Palavras & $M$ & $D P$ \\
\hline Sucesso & 2,58 & 1,74 & Tesão & 3,11 & 1,90 & Túmulo & 6,28 & 1,29 & Vidro & 6,31 & 1,07 \\
\hline Suco & 6,34 & 1,09 & Tinta & 6,38 & 1,16 & Unha & 6,65 & 0,84 & Vigilante & 4,80 & 1,80 \\
\hline Suculenta & 3,35 & 1,59 & Tirânico & 3,27 & 1,80 & Uniforme & 6,27 & 1,37 & Vingança & 2,56 & 1,39 \\
\hline Sufoco & 2,38 & 1,30 & Título & 3,79 & 1,98 & Urbanização & 4,41 & 1,84 & Violão & 6,74 & 1,02 \\
\hline Sujeira & 5,60 & 1,47 & Tomar & 3,80 & 1,80 & Ursinho & 6,27 & 1,32 & Violência & 3,79 & 1,89 \\
\hline Superfície & 4,78 & 1,76 & Torta & 6,15 & 1,40 & Usurpador & 2,37 & 1,42 & Virtude & 2,03 & 1,56 \\
\hline Superior & 2,73 & 1,59 & Tortura & 3,32 & 1,88 & Útil & 2,11 & 1,28 & Visão & 4,44 & 1,95 \\
\hline Suspiro & 3,64 & 1,92 & Tosse & 5,35 & 1,77 & Uva & 6,78 & 0,73 & Visita & 3,72 & 1,90 \\
\hline Sustentação & 2,60 & 1,54 & Trabalho & 4,47 & 1,78 & Vaca & 6,80 & 0,62 & Vitamina & 4,90 & 1,90 \\
\hline Susto & 3,24 & 1,82 & Tragar & 3,79 & 1,82 & Valoroso & 2,28 & 1,50 & Vitória & 2,54 & 1,60 \\
\hline Tabaco & 5,25 & 1,74 & Traição & 3,37 & 1,92 & Vantagem & 2,03 & 1,22 & Vomitar & 5,40 & 1,88 \\
\hline Tamanho & 3,30 & 1,70 & Tranqüilidade & 2,34 & 1,60 & Velocidade & 3,77 & 1,88 & Voz & 4,11 & 1,98 \\
\hline Tampa & 6,07 & 1,47 & Transar & 5,18 & 1,58 & Velório & 5,50 & 1,70 & Vulcão & 6,07 & 1,67 \\
\hline Tarde & 3,80 & 1,84 & Trânsito & 4,94 & 1,57 & Vendedor & 5,66 & 1,49 & Vulgaridade & 2,92 & 1,54 \\
\hline Tartaruga & 6,75 & 0,68 & Transporte & 5,01 & 1,62 & Veneno & 5,01 & 1,69 & Xadrez & 5,92 & 1,38 \\
\hline Tecido & 5,87 & 1,51 & Trauma & 2,49 & 1,58 & Veneziana & 5,32 & 2,05 & Xícara & 6,85 & 0,69 \\
\hline Teia & 5,90 & 1,47 & Travesseiro & 6,70 & 0,92 & Vento & 4,36 & 1,99 & Xingação & 2,42 & 1,46 \\
\hline Televisão & 6,85 & 0,62 & Tremor & 3,56 & 1,76 & Verde & 4,50 & 2,17 & Zeloso & 2,59 & 1,61 \\
\hline Temor & 2,06 & 1,41 & Tricô & 5,77 & 1,76 & Vermelho & 4,96 & 1,94 & Zombador & 3,07 & 1,58 \\
\hline Temperatura & 3,73 & 1,91 & Trigo & 6,20 & 1,47 & Vestir & 5,53 & 1,45 & Zoológico & 6,32 & 1,19 \\
\hline Tênis & 6,78 & 0,61 & Triste & 2,49 & 1,61 & Viagem & 4,62 & 1,74 & Zorra & 3,57 & 1,71 \\
\hline Tensão & 2,48 & 1,67 & Tristeza & 2,64 & 1,76 & Viajar & 4,27 & 1,84 & & & \\
\hline Terra & 5,94 & 1,66 & Trono & 6,13 & 1,41 & Vício & 3,19 & 1,64 & & & \\
\hline Terror & 2,37 & 1,39 & Tubarão & 6,76 & 0,79 & Vida & 2,96 & 2,06 & & & \\
\hline
\end{tabular}

Legenda: $M=$ concretude média, $D \mathrm{P}=$ desvio-padrão. 